\title{
HDAC6 regulates IL-17 expression in T lymphocytes: implications for HDAC6-targeted therapies
}

\author{
Bing Yan ${ }^{1,2^{*}}$, Yang $\mathrm{Liu}^{2 *}$, Hong Bai ${ }^{3}$, Miao Chen ${ }^{1}$, Songbo Xie ${ }^{1}$, Dengwen Li², Min Liu ${ }^{1}$, and Jun Zhou ${ }^{1,2}$ \\ 1. Key Laboratory of Animal Resistance Biology of Shandong Province, Institute of Biomedical Sciences, College of Life Sciences, Shandong Normal \\ University, Jinan, Shandong 250014, China. \\ 2. State Key Laboratory of Medicinal Chemical Biology, College of Life Sciences, Nankai University, Tianjin 300071, China. \\ 3. Key Laboratory of Immune Microenvironment and Disease of the Ministry of Education, School of Basic Medical Sciences, Tianjin Medical University, \\ Tianjin 300070, China. \\ *These authors contributed equally to this work.
}

$\square$ Corresponding author: Jun Zhou, Institute of Biomedical Sciences, College of Life Sciences, Shandong Normal University, 88 East Wenhua Road, Jinan, Shandong 250014, China. Telephone: +86-531-8618-2516; Fax: +86-531-8618-2518; E-mail: junzhou@sdnu.edu.cn.

(c) Ivyspring International Publisher. This is an open access article distributed under the terms of the Creative Commons Attribution (CC BY-NC) license (https://creativecommons.org/licenses/by-nc/4.0/). See http://ivyspring.com/terms for full terms and conditions.

Received: 2016.09.17; Accepted: 2017.01.03; Published: 2017.02.23

\begin{abstract}
The pro-inflammatory cytokine interleukin 17 (IL-17) is critically involved in immunity and inflammation. T-helper 17 and $\gamma \delta$ T cells are the predominant sources of IL-17 in the immune system. However, the mechanisms by which the expression of IL-17 is regulated in T cells remain elusive. Here, we demonstrate that loss of histone deacetylase 6 (HDAC6) in mice does not affect the generation of CD4+ or CD8+ $\mathrm{T}$ cells, but stimulates the development of IL-17-producing $\mathrm{Y} \delta \mathrm{T}$ cells. Our data further show that HDAC 6 deficiency increases the production of IL-17 by $\mathrm{V}_{\mathrm{Y}} 4^{+} \mathrm{V} \delta \mathrm{T}$ cells in the spleen and lymph nodes. Consistent with these observations, small-molecule inhibition of HDAC6 activity in $ү \delta T$ cells promotes the expression of IL-17 in vitro. These data thus reveal that HDAC6 represses IL-17 production in T cells, providing novel insights into the role of HDAC6 in the immune system. These findings also have important implications for the clinical investigation of HDAC6-targeted therapies.
\end{abstract}

Key words: histone deacetylase 6; interleukin 17; T cell; development; knockout mouse.

\section{Introduction}

Pro-inflammatory cytokines stimulate inflammation in response to pathogen infection, as well as in response to autoantigens in autoimmune disorders. Members of the interleukin 17 (IL-17) family play important roles in inflammation, in addition to acting as key players in the development of many autoimmune diseases [1-4]. IL-17 is expressed by both innate and adaptive immunocytes. As a part of the adaptive immune response, naïve $\mathrm{CD}^{+} \alpha \beta \mathrm{T}$ cells can differentiate into T-helper 17 (Th17) cells, which become mature in the peripheral organs via stimulation with specific cytokines [5, 6]. These cells are regarded as the primary source of IL-17 in autoimmune disorders.

With respect to the innate immune response, like Th17 cells, $\gamma \delta \mathrm{T}$ cells have been shown to produce IL-17A and IL-17F, two of the most well-characterized members of the IL-17 cytokine family [7]. Unlike Th17 cells, IL-17-producing $\gamma \delta \mathrm{T}$ cells differentiate during embryonic development and subsequently migrate into the mucous tissues after maturity, where they interact with outside pathogens. IL-17-producing $\gamma \delta \mathrm{T}$ cells residing in the spleen and lymph glands also appear to participate in innate inflammation. Expression of IL-17 by $\gamma \delta \mathrm{T}$ cells plays a protective role in concanavalin A-induced liver injury [8]. Although the precise signaling pathways that regulate IL-17 expression are not very well characterized, increasing evidence suggests that different mechanisms regulate the development of Th17 and IL-17-producing $\gamma \delta \mathrm{T}$ cells. For example, the transcription factor STAT3 (signal transducer and activator of transcription 3) is required for the development of Th17 cells, but does not appear to 
play a role in the development of IL-17-producing $\gamma \delta$ $\mathrm{T}$ cells $[9,10]$. However, little is known regarding the specific pathways that regulate IL-17 expression in $\gamma \delta$ T cells.

Histone deacetylase 6 (HDAC6) is unique among the HDACs because of its cytoplasmic localization and non-histone substrates. Several lines of evidence support the hypothesis that the deacetylase activity of HDAC6 serves as a key means for the regulation of the immune response [11]. HDAC6 plays a role in the organization of the immunological synapse through deacetylation of microtubules [12]. Furthermore, HDAC6 inhibition leads to hyperacetylation of Hsp90, thereby suppressing the activity of Foxp3 ${ }^{+} \mathrm{T}$ regulatory cells $[13,14]$. Additionally, we have previously demonstrated that HDAC6 regulates lipopolysaccharide-induced macrophage activation in a deacetylase activity-dependent manner [15]. In the present study, we have explored the role of HDAC6 in the regulation of IL-17 expression in T lymphocytes. Results from this study provide novel insight into the mechanisms that regulate IL-17 expression and further implicate HDAC6 in the immune response.

\section{Materials and Methods}

\section{Mice and cell culture}

HDAC6 heterozygous (HZ) mice were obtained from Tso-Pang Yao (Duke University, Durham, NC, USA) and intercrossed to generate HDAC6 knockout (KO) and wild-type (WT) littermates as described previously $[16,17]$. Mice were maintained in a specific pathogen-free barrier facility. All procedures for mouse care and operation were carried out according to the guidelines of experimental animals. The use of mice was approved by our Institutional Animal Care Committee. In vitro expansion of $\gamma \delta \mathrm{T}$ cells was performed as previously described [18]. EL4 murine lymphoma cells were cultured at $37^{\circ} \mathrm{C}$ in RPMI 1640 medium containing $10 \%$ fetal bovine serum.

\section{Flow cytometry}

Primary cells isolated from WT and HDAC6 KO mice were incubated with antibodies directed against specific cell surface molecules. The cells were then fixed, permeabilized, and stained with the indicated antibodies directed against intracellular antigens as previously described [19-21]. For flow cytometry analysis, $1 \times 10^{6}$ cells were resuspended in phosphate-buffered saline at a final concentration of 5 $\times 10^{5}$ cells $/ \mathrm{mL}$. Fluorescence was analyzed using a BD FACSCalibur instrument.

\section{Real-time quantitative reverse transcription- PCR (qRT-PCR)}

Total RNA was isolated from EL4 cells using the
TriPure isolation reagent (Roche) according to the manufacturer's protocol. cDNA was prepared using the M-MLV reverse transcriptase (Promega) and Oligo(dT) primers (Takara). The following specific primers were used for the PCR reactions: Hes1 forward: 5'-ACACCGGACAAACCAAAGAC-3', Hes1 reverse: 5'-ATGCCGGGAGCTATCTTTCT-3'; SOX13 forward: 5'-CCCTATTTCTCTCCAGACTGTT TCTT-3', SOX13 reverse: 5'-GCTGGTTAAGTTATTCA TCATTATCTTCTT-3'; SOX4 forward: 5'-GAACGC CTTTATGGTGTGGT-3', SOX4 reverse: 5'-GAACGG AATCTTGTCGCTGT-3'; IL-17A forward: 5'-TGAA GGCAGCAGCGATCA-3', IL-17A reverse: 5'-GGAAG TCCTTGGCCTCAGTGT-3'; IL-17F forward: 5'-CGC CATTCAGCAAGAAATCC-3', IL-17F reverse: 5'-CTC CAACCTGAAGGAATTAGAACAG-3'. $\beta$-actin served as a reference gene for normalization.

\section{Luciferase assays}

Cells harvested from a $10-\mathrm{cm}$ culture dish were transfected by electroporation with a pGL3 firefly luciferase reporter plasmid containing the IL-17 promoter sequence. Cells were co-transfected with the pRL-TK renilla luciferase reporter plasmid to control for the transfection efficiency. Luciferase activity was measured, and luciferase activity was reported as the firefly/renilla luciferase ratio as described [22, 23].

\section{Statistics}

Analysis of statistical significance was performed by the Student's t-test for comparison between two groups and by the ANOVA test for multiple comparisons.

\section{Results}

\section{Loss of HDAC6 does not affect the development of $\mathrm{CD4}^{+}$or $\mathrm{CD8}^{+} \mathrm{T}$ lymphocytes}

Although several reports have revealed an important role for HDAC6 in T lymphocyte function, a link between HDAC6 and the development of T lymphocytes remains largely unknown. We explored the role of HDAC6 in the development of $\mathrm{T}$ lymphocytes by analyzing the populations of $\mathrm{CD}^{+}$ and $\mathrm{CD}^{+} \mathrm{T}$ lymphocytes in both lymphoid and non-lymphoid tissues using flow cytometry. In agreement with the previous study, HDAC6 deficiency had no obvious effect on the development of $\mathrm{CD}^{+} / \mathrm{CD}^{+}, \mathrm{CD}^{+}$, or $\mathrm{CD} 8^{+} \mathrm{T}$ lymphocytes in the thymus (Figure 1A-D). Furthermore, the loss of HDAC6 did not significantly alter the distribution of the $\mathrm{CD}^{+}$or $\mathrm{CD} 8^{+} \mathrm{T}$ lymphocytes in peripheral tissues, including the spleen, lymph nodes, liver, and blood (Figure 1A-D). 
A
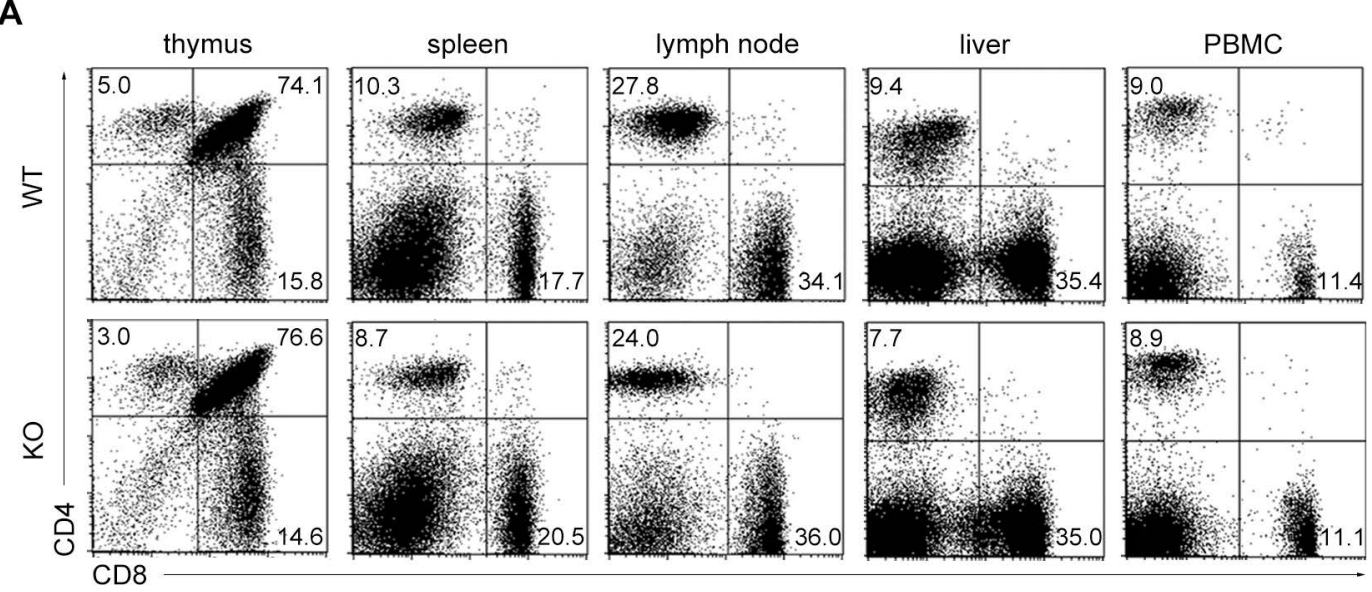

B

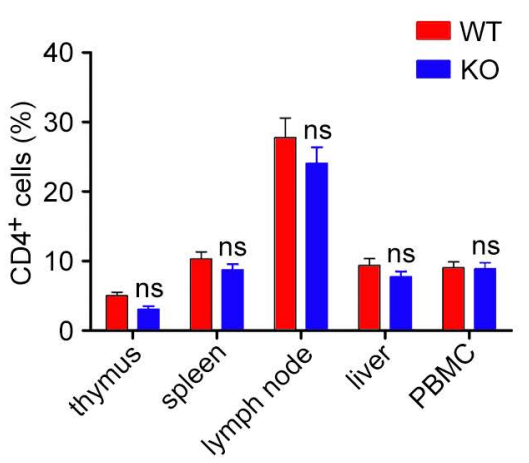

C

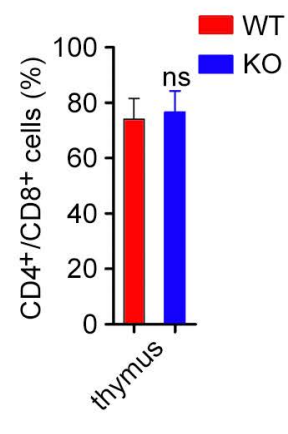

D

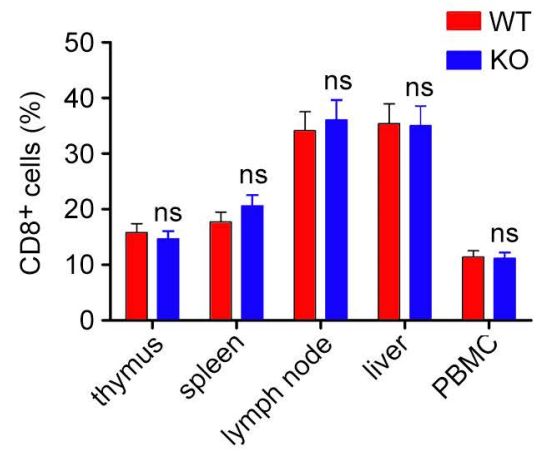

Figure 1. HDAC6 deficiency does not affect the development of CD4+ or CD8 ${ }^{+} \mathrm{T}$ cells. (A) Primary lymphocytes were isolated from the thymus, spleen, lymph nodes, liver, and PBMC (peripheral blood mononuclear cells) of WT and HDAC6 KO mice. CD4 ${ }^{+}, \mathrm{CD}^{+}$, and $\mathrm{CD}^{+} / \mathrm{CD}^{+}$subpopulations of T cells (selected by gating $\mathrm{CD}^{+}$cells) were analyzed by flow cytometry. (B-D) Quantification of the percentages of CD4+ $(B), \mathrm{CD}^{+} / \mathrm{CD} 8^{+}(\mathrm{C})$, and CD8+ $(\mathrm{D})$ cells in WT and HDAC6 $\mathrm{KO}$ mice based on two-color flow cytometric analysis as shown in (A). Data are representative of three independent experiments. Error bars indicate mean $\pm \mathrm{SEM}$. ns, not significant.

\section{HDAC6 deficiency results in an increase in the population of IL-17-producing Y $\delta \mathrm{T}$ cells}

We next compared the IL-17-producing and interferon- $\gamma($ IFN- $\gamma)$-producing subpopulations of $\gamma \delta$ $\mathrm{T}$ and $\mathrm{CD}^{+} \mathrm{T}$ cells. Flow cytometry revealed that the number of IL-17-producing CD4 ${ }^{+} \mathrm{T}$ cells in the spleen was slightly increased in HDAC6 KO mice, while the number of IL-17-producing $\gamma \delta \mathrm{T}$ cells was dramatically increased in HDAC6 KO mice (Figure $2 \mathrm{~A}$ and $\mathrm{B})$. In contrast, the number of IFN- $\gamma$-producing $\gamma \delta \mathrm{T}$ and $\mathrm{CD}^{+} \mathrm{T}$ cells was not significantly affected by HDAC6 deficiency (Figure $2 \mathrm{~A}$ and $\mathrm{B})$. These results suggest a key role for HDAC6 in the regulation of IL-17 production by $\gamma \delta \mathrm{T}$ cells.

We next analyzed the subpopulations of IL-17-producing $\gamma \delta \mathrm{T}$ cells by staining with anti-V $\gamma 1$ or anti- $\mathrm{V}_{\gamma} 4$ antibodies. Consistent with previous studies, our results showed that the ${\mathrm{V} \gamma 4^{+}}^{+}$subset represented the major source of IL-17-producing $\gamma \delta \mathrm{T}$ cells in both spleens and lymph glands (Figure 2C and D). Deletion of HDAC6 also increased IL-17 production in these ${\mathrm{V} \gamma 4^{+}}^{+} \delta$ T cells (Figure 2C and D).
The deacetylase activity of HDAC6 is required for its regulation of IL- 17 production by $\mathrm{Y} \delta \mathrm{T}$ cells

We then sought to determine whether the deacetylase activity of HDAC6 is involved in its regulation of IL-17 production by $\gamma \delta \mathrm{T}$ cells. We isolated $\gamma \delta \mathrm{T}$ cells from the spleen of mice and expanded the cells in vitro. Consistent with the in vivo findings, treatment of the in vitro expanded $\gamma \delta \mathrm{T}$ cells with tubacin, a selective inhibitor of HDAC6 [24], significantly increased IL-17 production (Figure 3A and B). Interestingly, deletion or inhibition of HDAC6 decreased the amount of IFN- $\gamma$-producing $\gamma \delta \mathrm{T}$ cells in vitro (Figure $3 \mathrm{~A}$ and $\mathrm{C}$ ), which was not significantly affected by HDAC6 deficiency in vivo (Figure 2A and B), reflecting the different sensitivity of this subpopulation of $\gamma \delta \mathrm{T}$ cells to HDAC6 deficiency in the in vitro and in vivo systems. Collectively, these results indicate that the role of HDAC6 in the regulation of IL-17 production by $\gamma \delta \mathrm{T}$ cells is dependent on its deacetylase activity. 
A

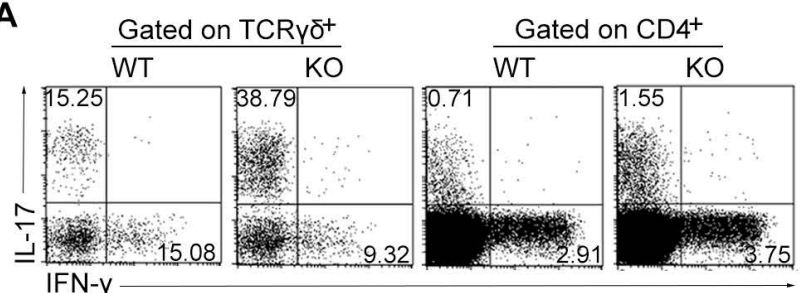

B

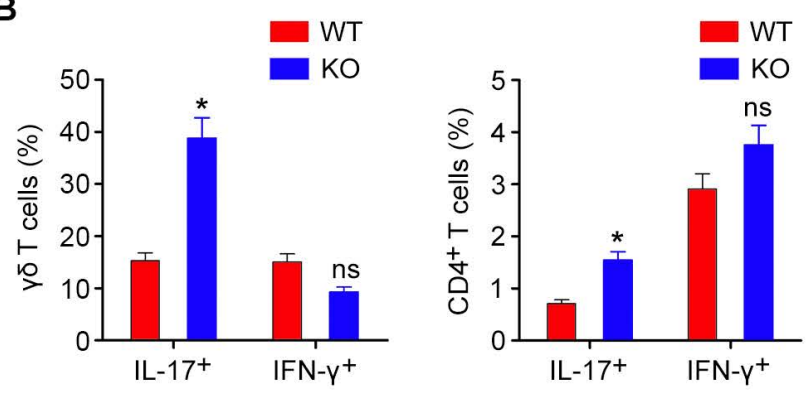

C
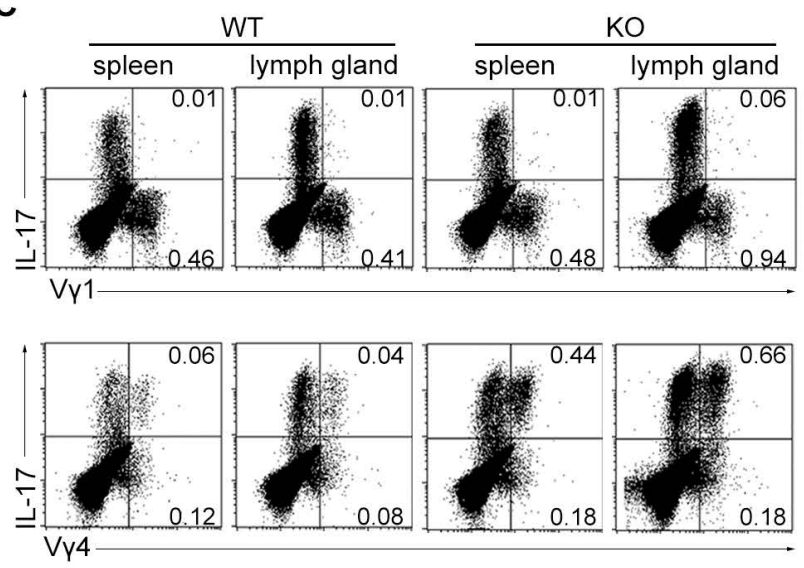

D

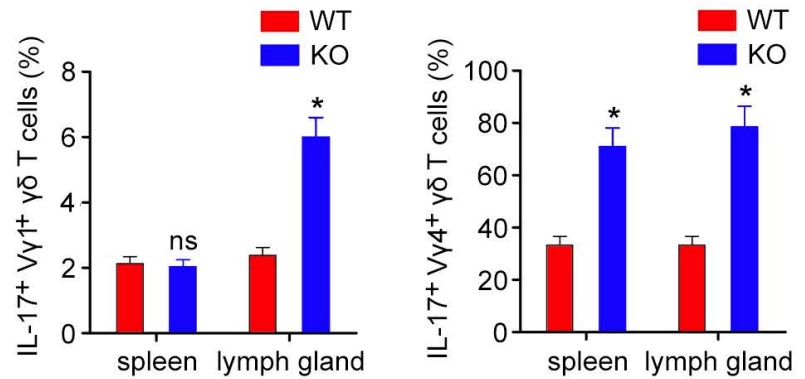

Figure 2. HDAC6 deficiency increases the development of IL-17-producing $y \delta$ T cells. (A) Lymphocytes isolated from the spleen of WT and HDAC6 KO mice were stimulated with PMA and ionomycin, and the expression of IFN- $\gamma$ and IL-17 was analyzed in $\gamma \delta$ T and CD4+ T cells. (B) Quantification of the IFN- $\gamma$ and IL-17-producing subpopulations of $\mathrm{y} \delta \mathrm{T}$ and $\mathrm{CD} 4^{+} \mathrm{T}$ cells based on flow cytometry as shown in (A). (C) Lymphocytes isolated from the spleen or lymph glands of WT and HDAC6 KO mice were stimulated with PMA and ionomycin, and the expression of $\mathrm{IL}-17$ in $\mathrm{VY}^{1+}$ or $\mathrm{V} 4^{+} \mathrm{Y} \delta \mathrm{T}$ cells was analyzed by gating $\mathrm{CD3}^{+}$cells. (D) Quantification of IL-17-producing subpopulations of $\mathrm{VYI}^{+}$or $\mathrm{V} Y 4^{+} \mathrm{y} \delta \mathrm{T}$ cells based on flow cytometry as shown in (C). Data are representative of three independent experiments. Error bars indicate mean \pm SEM. ${ }^{*} p<0.05 ;$ ns, not significant.
HDAC6 deficiency promotes the development of IL-17-producing $Y \delta T$ cells in the thymus

Development of $\gamma \delta$ T cells primarily occurs in the thymus during mouse embryonic development. To assess the role of HDAC6 in the development of $\gamma \delta \mathrm{T}$ cells in the thymus, we isolated thymocytes from newborn WT, HDAC6 HZ, and HDAC6 KO mice. Immunostaining and flow cytometric analysis revealed that the levels of thymic IL-17-producing $\gamma \delta$ $\mathrm{T}$ cells were increased in HDAC6 $\mathrm{HZ}$ and $\mathrm{KO}$ mice (Figure 4A and B). These results, together with the data shown above (Figure 3), suggest that HDAC6 may regulate the fate of $\gamma \delta \mathrm{T}$ cells by determining whether they differentiate into IL-17-producing or IFN- $\gamma$-producing cells.
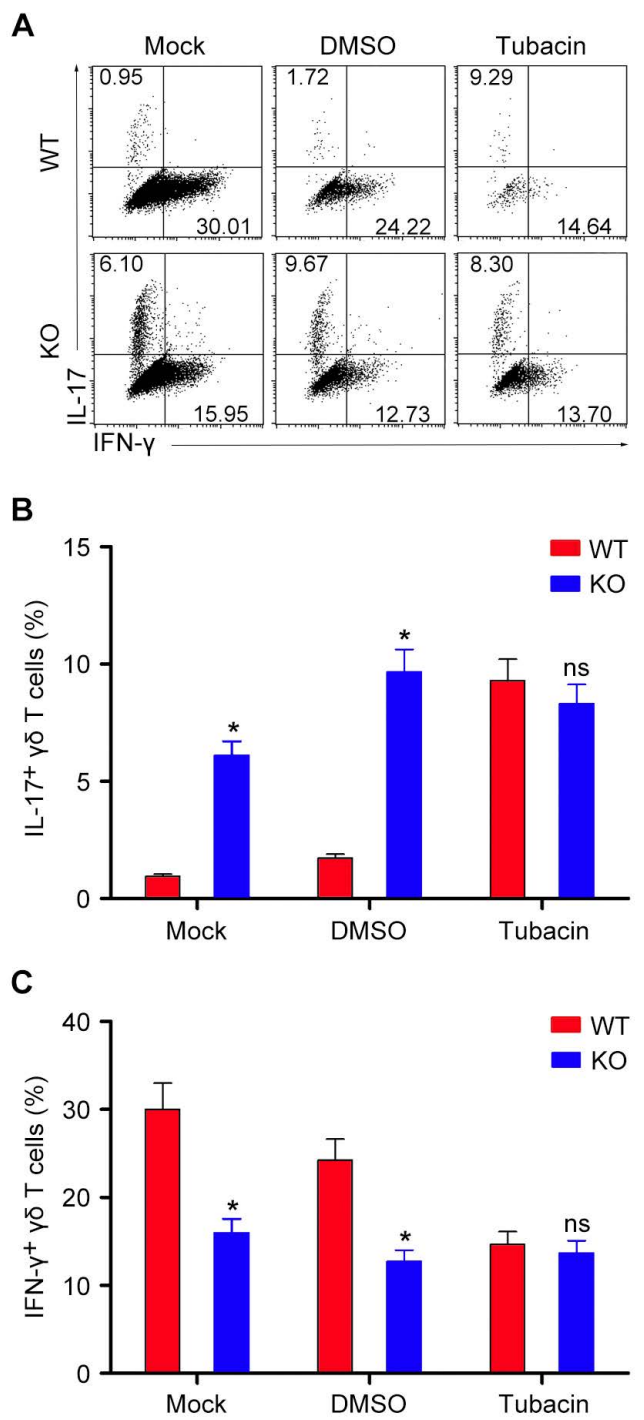

Figure 3. The deacetylase activity of HDAC6 is required for its regulation of IL-17 production by $Y \delta$ T cells. (A) $y \delta T$ cells isolated from the spleen of WT and HDAC6 KO mice were cultured for 6 days and treated with $1 \mu \mathrm{M}$ tubacin or an equal volume of the vehicle DMSO. IFN-Y- and IL-17-producing subpopulations of $y \delta T$ cells were then analyzed by flow cytometry. ( $B$ and $C$ ) Quantification of the percentages of IL-17-producing (B) and IFN-Y-producing (C) $ү \delta ~ T$ cells. Data are representative of three independent experiments. Error bars indicate mean \pm SEM. $* p<0.05$; ns, not significant. 


\section{HDAC6 regulates IL-17A and IL-17F mRNA expression}

To examine whether HDAC6 regulates IL-17 mRNA expression in $\gamma \delta$ T cells, we stimulated the in vitro expanded $\gamma \delta \mathrm{T}$ cells with PMA and ionomycin and then analyzed the levels of the IL-17A and IL-17F mRNAs by real-time qRT-PCR. We found that the mRNA levels of both IL-17A and IL-17F were remarkably increased upon the loss of HDAC6 in $\gamma \delta \mathrm{T}$ cells (Figure 5A).

To confirm the effect of HDAC6 on IL-17 expression, we used EL4 murine lymphoma cells, which have been employed as a model to study IL-17 production by T cells [25]. EL4 cells were stimulated with PMA and ionomycin, and the levels of the IL-17A and IL-17F mRNAs were quantified by real-time qRT-PCR. Stimulation of EL4 cells with PMA and ionomycin increased the expression of IL-17A and IL-17F (Figure 5B). Therefore, we next explored the role of HDAC6 in the regulation of IL-17A and IL-17F transcription in activated EL4 cells by treating cells with the HDAC6 inhibitor tubacin. We found that tubacin increased both IL-17A and IL-17F expression in a concentration-dependent manner (Figure 5C and D). Similar results were achieved with tubastatin A (Figure S1), another HDAC6-selective inhibitor [26].
A

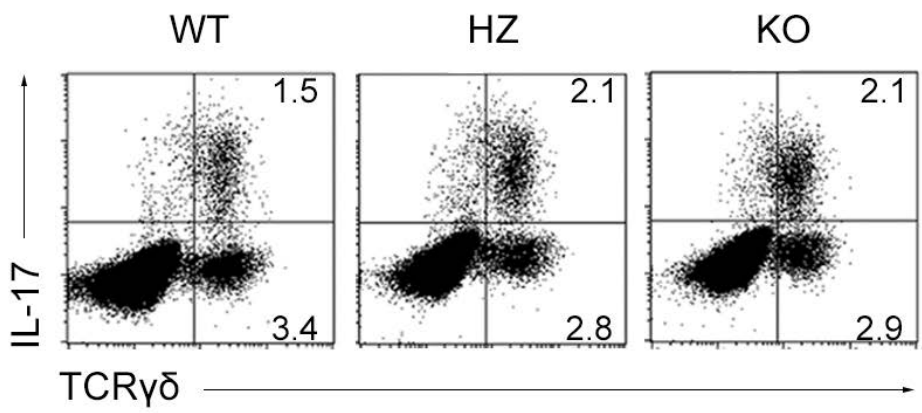

B

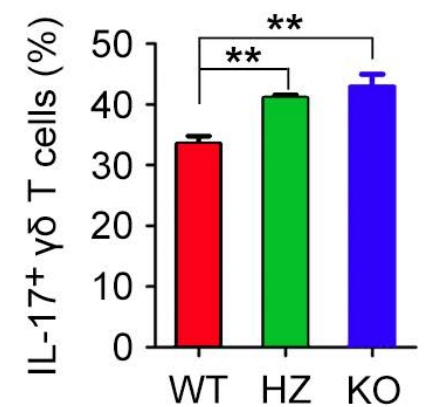

Figure 4. HDAC6 regulates the development of IL-17-producing $\gamma \delta T$ cells in the thymus. (A) Thymic lymphocytes were isolated from newborn WT, HDAC6 HZ, and HDAC6 KO mice. IL-17 expression in $ү \delta \mathrm{T}$ cells was analyzed by flow cytometry after stimulation with PMA and ionomycin. (B) Quantification of the percentage of IL-17-producing $y \delta T$ cells in WT, HDAC6 HZ, and HDAC6 KO mice. Data are representative of three independent experiments. Error bars indicate mean \pm SEM. $* * p<0.01$.

A

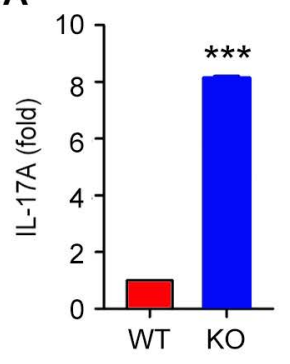

C
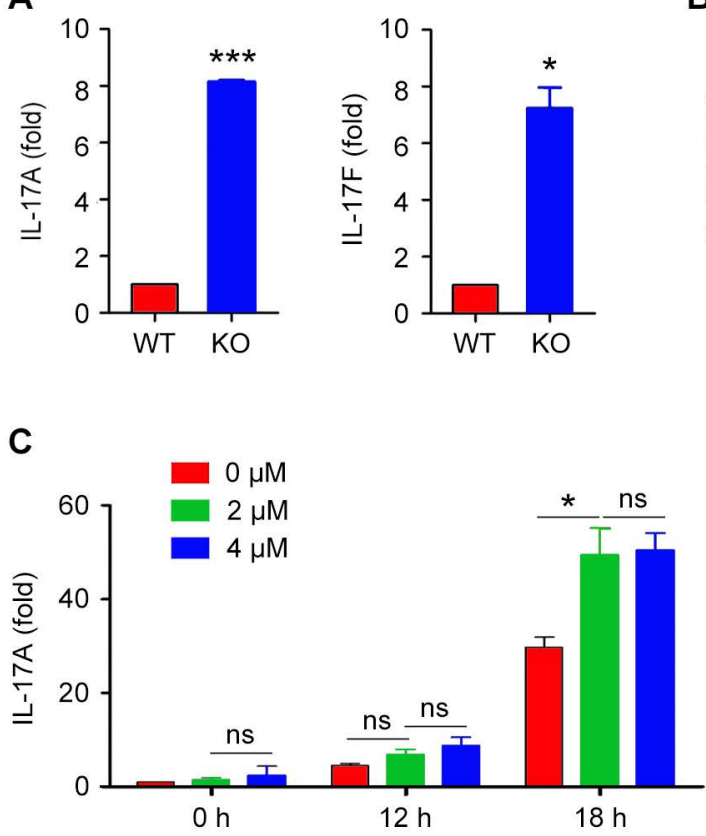

B
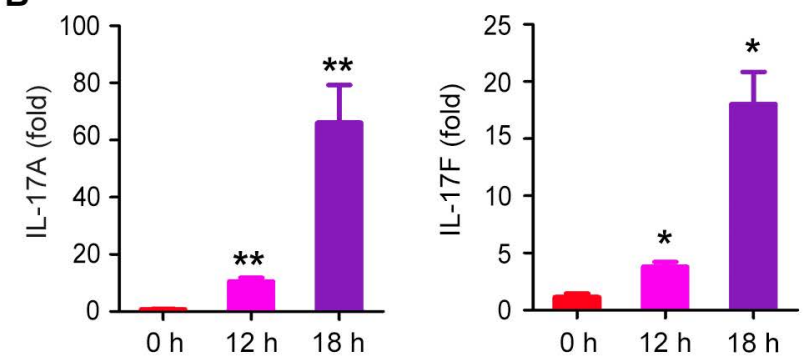

D

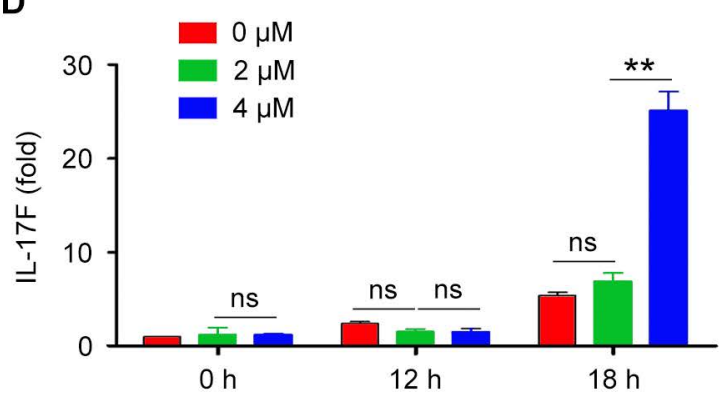

Figure 5. Effects of HDAC6 on IL-17 mRNA expression. (A) qRT-PCR analysis of IL-17A and IL-17F mRNA levels in WT and HDAC6 KO үס T cells expanded in vitro for 6 days and incubated with PMA and ionomycin for 6 hours. (B) qRT-PCR analysis of IL-17A and IL-17F mRNA levels in EL4 cells incubated with PMA and ionomycin for 0,12 , or 18 hours. (C and D) qRT-PCR analysis of IL-17A and IL-17F mRNA levels in EL4 cells treated with 0 , 2 , or $4 \mu M$ tubacin for 4 hours, followed by incubation with PMA and ionomycin for 0,12 , or 18 hours. Data are representative of three independent experiments. Error bars indicate mean \pm SEM. $* p<0.05$; $* * p<0.01 ; * * * p<0.001 ;$ ns, not significant. 


\section{Effects of HDAC6 on IL-17-associated transcription factors and IL-17 promoter-dependent luciferase activity}

Hes1, SOX4, and SOX13 are key regulators for the development of IL-17-producing $\gamma \delta \mathrm{T}$ cells. To determine whether these transcription factors are involved in IL-17 production, we analyzed the levels of Hes1, SOX4, and SOX13 mRNAs using real-time qRT-PCR. We found that the loss of HDAC6 decreased the expression of SOX 4 in $\gamma \delta$ T cells, but did not significantly affect the expression of Hes1 or SOX13 (Figure 6A). PMA and ionomycin decreased the expression of these transcription factors in EL4 cells (Figure 6B). Inhibition of HDAC6 with tubacin or tubastatin A did not rescue Hes1, SOX4, and SOX13 expression (Figure 6C and Figure S2). These results suggest that alternative mechanisms may underlie the regulation of IL-17 production by HDAC6.

We next analyzed whether the thymus-specific isoform of retinoic acid receptor-related orphan receptor $\gamma(R O R \gamma t)$, a transcription factor critical for the differentiation program of pro-inflammatory Th17 cells [27], is involved in the regulation of IL-17 production. Activity of the firefly luciferase under control of the IL-17 promoter could be increased by the expression of RORyt alone (Figure 6D). Similarly, increased RORyt expression also increased IL-17 promoter-dependent luciferase activity (Figure 6E). Furthermore, consistent with our findings that HDAC6 represses IL-17 expression in $\gamma \delta \mathrm{T}$ cells, inhibition of HDAC6 with tubacin also significantly increased the IL-17 promoter-dependent luciferase activity (Figure 6F).
A
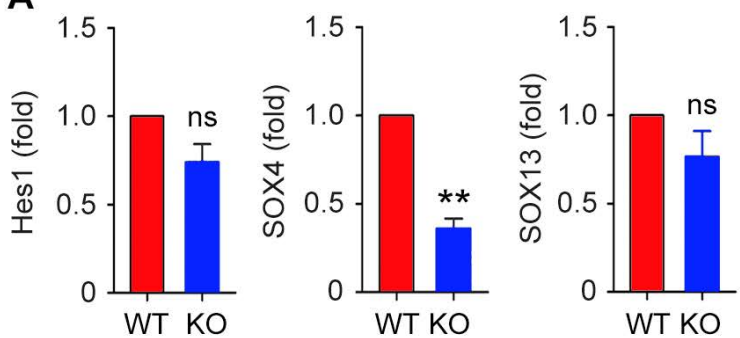

B
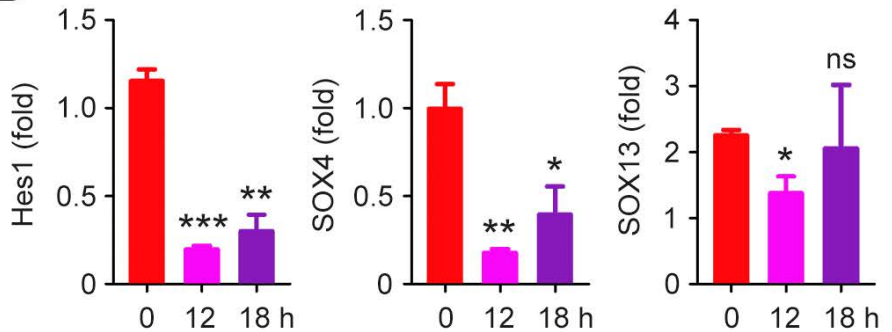
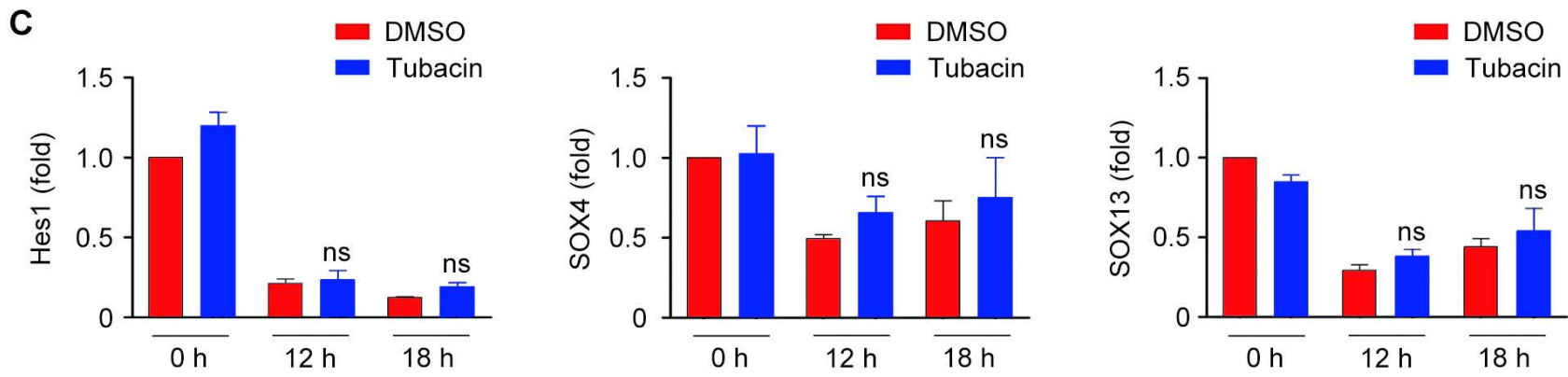

D

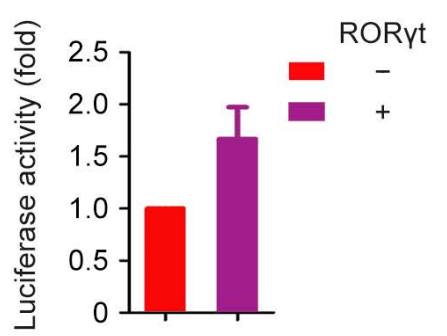

$\mathbf{E}$

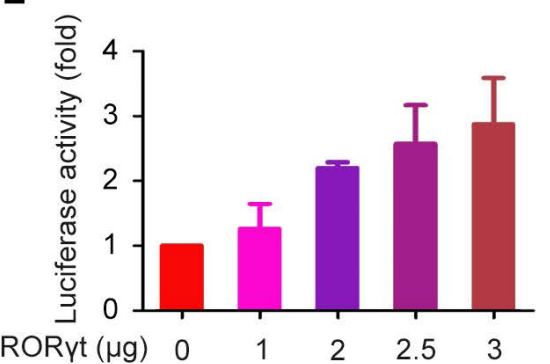

$\mathbf{F}$

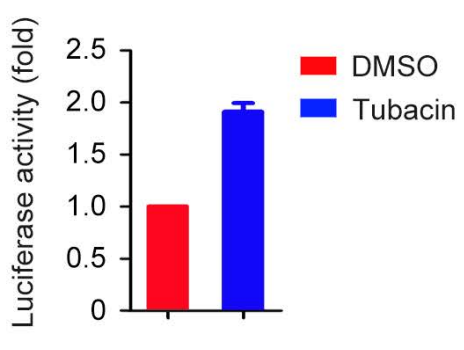

Figure 6. Effects of HDAC6 on IL-17-associated transcription factors and IL-17 promoter-dependent luciferase activity. (A) qRT-PCR analysis of Hes I, SOX4, and SOX13 mRNA levels in WT and HDAC6 KO Y $\delta \mathrm{T}$ cells expanded in vitro for 6 days and incubated with PMA and ionomycin for 6 hours. (B) qRT-PCR analysis of Hes 1 , SOX4, and SOX13 mRNA levels in EL4 cells incubated with PMA and ionomycin for 0, 12, or 18 hours. (C) qRT-PCR analysis of Hes 1, SOX4, and SOX13 mRNA levels in EL4 cells treated with DMSO or $2 \mu \mathrm{M}$ tubacin for 4 hours, followed by incubation with PMA and ionomycin for 0 , 12 , or 18 hours. (D) Quantification of the relative luciferase activity in cells transfected with the firefly luciferase reporter under control of the IL-17 promoter, along with an RORYt expression plasmid. (E) Quantification of the relative luciferase activity in EL4 cells transfected with the firefly luciferase reporter under control of the IL-17 promoter, together with increasing amounts of the RORyt expression plasmid. (F) Quantification of the relative luciferase activity in EL4 cells transfected with the firefly luciferase reporter under control of the IL-17 promoter and treated with DMSO or $2 \mu \mathrm{M}$ tubacin. Error bars indicate mean \pm SEM. $*_{p}<0.05$; $* *<<0.01$; $* * * p<0.001$; ns, not significant. 


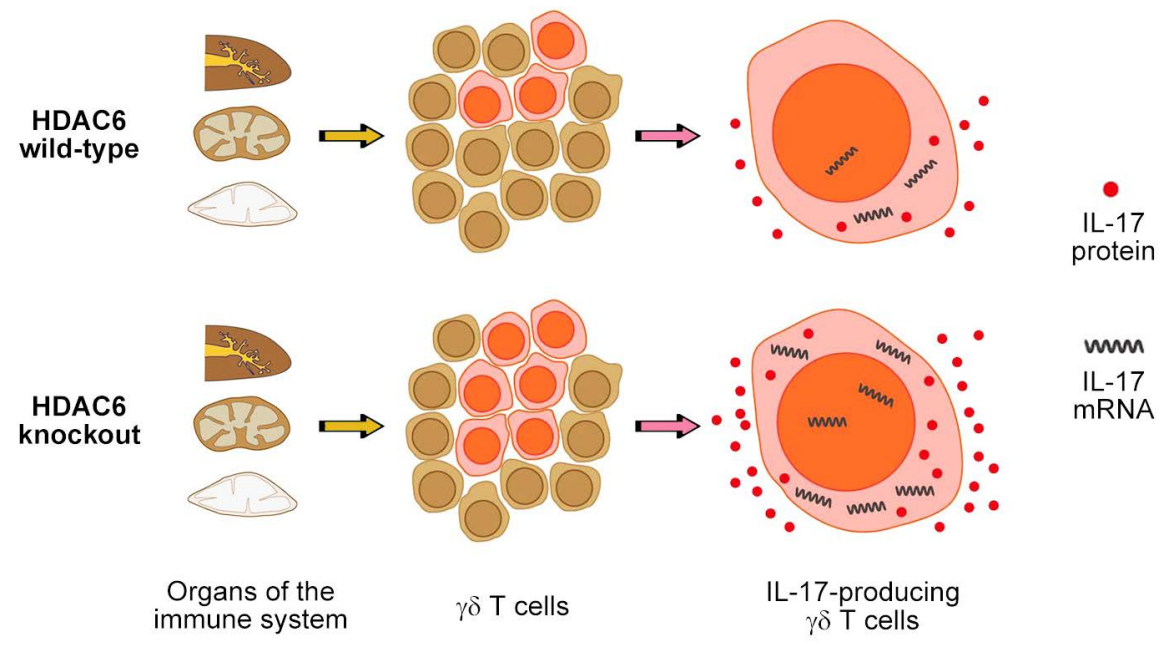

Figure 7. A working model for HDAC6 function in the regulation of IL-17 expression in $\gamma \delta$ T cells.

\section{Discussion}

Our study demonstrates that deletion of HDAC6 in mice or inhibition of its deacetylase activity promotes IL-17 expression in $\gamma \delta \mathrm{T}$ cells (Figure 7). Although a comprehensive understanding of the mechanisms underlying the regulation of IL-17 expression in distinct subsets of $\mathrm{T}$ cells remains elusive, our findings demonstrate that HDAC6 plays a crucial role in the regulation of IL-17 expression (Figure 7).

Considering the similar role of HDAC6 in the regulation of IL-17 expression in $\gamma \delta \mathrm{T}$ and EL4 cells examined in this study, we propose that the underlying mechanism is likely to be conserved and could potentially overlap known pathways by which HDAC6 regulates gene expression. For example, HDAC6 has previously been reported to play a role in the regulation of $\beta$-catenin and extracellular signal-regulated kinase (ERK) signaling pathways [28-30], as well as in the inactivation of Runt-related transcription factor 2 (Runx2), which has been shown to promote the expression of IL-17 [31]. These studies provide a plausible mechanism to explain the effect of HDAC6 activity on IL-17 expression that should be explored in future studies.

HDAC6-selective inhibitors are currently under investigation in multiple clinical settings, including cancer and neurodegenerative diseases [11, 32-34]. Our findings suggest that the side effects of HDAC6 inhibition on immunity should be considered as a part of clinical trials. Both IL-17A and IL-17F are known to play protective roles in the host defense, and $\mathrm{T}$ lymphocytes expressing IL-17 may recruit neutrophils to tissues undergoing inflammation [35]. Thus, increased IL-17A and IL-17F expression induced by HDAC6 inhibition may promote the clearance of extracellular bacterial or fungal infections. However, in addition to these pro-inflammatory roles of IL-17 in host immune responses, increased IL-17 production could also result in elevated risk for autoimmune diseases.

Results from this study demonstrate that loss of HDAC6 promotes the accumulation of IL-17-producing $\gamma \delta \mathrm{T}$ cells. This subset of $\mathrm{T}$ lymphocytes has previously been reported to play a protective role in concanavalin A-induced hepatitis [8]. Thus, it is possible that inhibition of HDAC6 could promote the differentiation of IL-17-producing $\gamma \delta \mathrm{T}$ cells during embryonic development, resulting in an increase in the population of IL-17-producing $\gamma \delta \mathrm{T}$ cells in peripheral immune organs. These results suggest that drugs targeting HDAC6 might be investigated for a protective role in hepatitis.

\section{Supplementary Material}

Supplementary figures.

http://www.thno.org/v07p1002s1.pdf

\section{Abbreviations}

IL-17, interleukin 17; Th17, T-helper 17; HDAC6, histone deacetylase 6; WT, wild-type; HZ, heterozygous; KO, knockout; qRT-PCR, quantitative reverse transcription-PCR; IFN- $\gamma$, interferon- $\gamma$; ROR $\gamma \mathrm{t}$, thymus-specific isoform of retinoic acid receptor-related orphan receptor $\gamma$; ERK, extracellular signal-regulated kinase; Runx2, Runt-related transcription factor 2.

\section{Acknowledgements}

We thank Tso-Pang Yao for providing HDAC6 heterozygous mice and Zhinan Yin and Guangchao Cao for discussion. This work was supported by grants from the National Natural Science Foundation 
of China (31471262 and 31671403) and the Tianjin Natural Science Foundation (15JCYBJC49300).

\section{Competing Interests}

The authors have declared that no competing interest exists.

\section{References}

1. Nakae S, Nambu A, Sudo K, Iwakura Y. Suppression of immune induction of collagen-induced arthritis in IL-17-deficient mice. J Immunol. 2003; 171: 6173-7.

2. Komiyama Y, Nakae S, Matsuki T, Nambu A, Ishigame H, Kakuta S, et al. IL-17 plays an important role in the development of experimental autoimmune encephalomyelitis. J Immunol. 2006; 177: 566-73.

3. Lohr J, Knoechel B, Wang JJ, Villarino AV, Abbas AK. Role of IL-17 and regulatory T lymphocytes in a systemic autoimmune disease. J Exp Med. 2006; 203: 2785-91.

4. Beringer $\mathrm{A}$, Noack $\mathrm{M}$, Miossec P. IL-17 in chronic inflammation: from discovery to targeting. Trends Mol Med. 2016; 22: 230-41.

5. Stockinger B, Veldhoen M. Differentiation and function of Th17 T cells. Curr Opin Immunol. 2007; 19: 281-6.

6. Furuzawa-Carballeda J, Vargas-Rojas MI, Cabral AR. Autoimmune inflammation from the Th17 perspective. Autoimmun Rev. 2007; 6: 169-75.

7. Chien $\mathrm{YH}$, Zeng $\mathrm{X}$, Prinz I. The natural and the inducible: interleukin (IL)-17-producing gammadelta T cells. Trends Immunol. 2013; 34: 151-4.

8. Zhao N, Hao J, Ni Y, Luo W, Liang R, Cao G, et al. Vgamma4 gammadelta T cell-derived IL-17A negatively regulates NKT cell function in Con A-induced fulminant hepatitis. J Immunol. 2011; 187: 5007-14.

9. Yang XO, Panopoulos AD, Nurieva R, Chang SH, Wang D, Watowich SS, et al. STAT3 regulates cytokine-mediated generation of inflammatory helper T cells. J Biol Chem. 2007; 282: 9358-63.

10. Shibata K, Yamada H, Sato T, Dejima T, Nakamura M, Ikawa T, et al. Notch-Hes1 pathway is required for the development of IL-17-producing gammadelta T cells. Blood. 2011; 118: 586-93.

11. Zhang L, Ogden A, Aneja R, Zhou J. Diverse roles of HDAC6 in viral infection: Implications for antiviral therapy. Pharmacol Ther. 2016; 164: 120-5.

12. Serrador JM, Cabrero JR, Sancho D, Mittelbrunn M, Urzainqui A, Sanchez-Madrid F. HDAC6 deacetylase activity links the tubulin cytoskeleton with immune synapse organization. Immunity. 2004; 20: 417-28

13. de Zoeten EF, Wang L, Butler K, Beier UH, Akimova T, Sai H, et al. Histone deacetylase 6 and heat shock protein 90 control the functions of Foxp3(+) T-regulatory cells. Mol Cell Biol. 2011; 31: 2066-78.

14. Beier UH, Wang L, Han R, Akimova T, Liu Y, Hancock WW. Histone deacetylases 6 and 9 and sirtuin- 1 control Foxp3+ regulatory T cell function through shared and isoform-specific mechanisms. Sci Signal. 2012; 5: ra45.

15. Yan B, Xie S, Liu Z, Ran J, Li Y, Wang J, et al. HDAC6 deacetylase activity is critical for lipopolysaccharide-induced activation of macrophages. PLoS One. 2014; 9: e110718.

16. Gao YS, Hubbert CC, Lu J, Lee YS, Lee JY, Yao TP. Histone deacetylase 6 regulates growth factor-induced actin remodeling and endocytosis. Mol Cell Biol. 2007; 27: 8637-47.

17. Zhang L, Liu S, Liu N, Zhang Y, Liu M, Li D, et al. Proteomic identification and functional characterization of MYH9, Hsc70, and DNAJA1 as novel substrates of HDAC6 deacetylase activity. Protein Cell. 2015; 6: 42-54.

18. He W, Hao J, Dong S, Gao Y, Tao J, Chi H, et al. Naturally activated V gamma 4 gamma delta $\mathrm{T}$ cells play a protective role in tumor immunity through expression of eomesodermin. J Immunol. 2010; 185: 126-33.

19. Liu M, Li D, Sun L, Chen J, Sun X, Zhang L, et al. Modulation of Eg5 activity contributes to mitotic spindle checkpoint activation and Tat-mediated apoptosis in CD4-positive T-lymphocytes. J Pathol. 2014; 233: 138-47.

20. Yang Y, Ran J, Sun L, Sun X, Luo Y, Yan B, et al. CYLD regulates noscapine activity in acute lymphoblastic leukemia via a microtubule-dependent mechanism. Theranostics. 2015: 5: 656-66.

21. Yin Z, Zhang DH, Welte T, Bahtiyar G, Jung S, Liu L, et al. Dominance of IL-12 over IL-4 in gamma delta $T$ cell differentiation leads to default production of IFN-gamma: failure to down-regulate IL-12 receptor beta 2-chain expression. J Immunol. 2000; 164: 3056-64.

22. Huo L, Li D, Sun L, Liu M, Shi X, Sun X, et al. Tat acetylation regulates its actions on microtubule dynamics and apoptosis in T lymphocytes. J Pathol. 2011; 223: 28-36

23. Huo L, Li D, Sun X, Shi X, Karna P, Yang W, et al. Regulation of Tat acetylation and transactivation activity by the microtubule-associated deacetylase HDAC6. J Biol Chem. 2011; 286: 9280-6.

24. Haggarty SJ, Koeller KM, Wong JC, Grozinger CM, Schreiber SL. Domain-selective small-molecule inhibitor of histone deacetylase 6 (HDAC6)-mediated tubulin deacetylation. Proc Natl Acad Sci U S A. 2003; 100: 4389-94.

25. Ichiyama K, Yoshida H, Wakabayashi Y, Chinen T, Saeki K, Nakaya M, et al. Foxp3 inhibits RORgammat-mediated IL-17A mRNA transcription through direct interaction with RORgammat. J Biol Chem. 2008; 283: 17003-8.
26. Butler KV, Kalin J, Brochier C, Vistoli G, Langley B, Kozikowski AP. Rational design and simple chemistry yield a superior, neuroprotective HDAC6 inhibitor, tubastatin A. J Am Chem Soc. 2010; 132: 10842-6.

27. Ivanov, II, McKenzie BS, Zhou L, Tadokoro CE, Lepelley A, Lafaille JJ, et al. The orphan nuclear receptor RORgammat directs the differentiation program of proinflammatory IL-17+ T helper cells. Cell. 2006; 126: 1121-33.

28. Li Y, Zhang X, Polakiewicz RD, Yao TP, Comb MJ. HDAC6 is required for epidermal growth factor-induced beta-catenin nuclear localization. J Biol Chem. 2008; 283: 12686-90.

29. Mak AB, Nixon AM, Kittanakom S, Stewart JM, Chen GI, Curak J, et al. Regulation of CD133 by HDAC6 promotes beta-catenin signaling to suppress cancer cell differentiation. Cell Rep. 2012; 2: 951-63.

30. Williams KA, Zhang M, Xiang S, Hu C, Wu JY, Zhang S, et al. Extracellular signal-regulated kinase (ERK) phosphorylates histone deacetylase 6 (HDAC6) at serine 1035 to stimulate cell migration. J Biol Chem. 2013; 288: 33156-70.

31. Ozaki T, Wu D, Sugimoto H, Nagase H, Nakagawara A. Runt-related transcription factor 2 (RUNX2) inhibits p53-dependent apoptosis through the collaboration with HDAC6 in response to DNA damage. Cell Death Dis. 2013; 4: e610.

32. Yu F, Ran J, Zhou J. Ciliopathies: does HDAC6 represent a new therapeutic target? Trends Pharmacol Sci. 2016; 37: 114-9.

33. Simoes-Pires C, Zwick V, Nurisso A, Schenker E, Carrupt PA, Cuendet M. HDAC6 as a target for neurodegenerative diseases: what makes it different from the other HDACs? Mol Neurodegener. 2013; 8: 7.

34. Seidel C, Schnekenburger M, Dicato M, Diederich M. Histone deacetylase 6 in health and disease. Epigenomics. 2015; 7: 103-18.

35. Shibata K, Yamada H, Hara H, Kishihara K, Yoshikai Y. Resident Vdelta1+ gammadelta $\mathrm{T}$ cells control early infiltration of neutrophils after Escherichia coli infection via IL-17 production. J Immunol. 2007; 178: 4466-72. 